\title{
Does momentary accessibility influence metacomprehension judgments? The influence of study-judgment lags on accessibility effects
}

\author{
JULIE M. C. BAKER and JOHN DUNLOSKY \\ Kent State University, Kent, Ohio
}

\begin{abstract}
In two experiments, we investigated momentary accessibility as a basis for metacomprehension judgments. Momentary accessibility has been cited as a major contributor to these judgments, yet the only previous investigation on the topic used judgments that were delayed a day after study, which have not been used in any other studies in the field and may be necessary for demonstrating accessibilitybased effects. As expected, Experiment 1 demonstrated that the time between study and judgments moderates accessibility effects, with the relationship between judgments and access measures being substantially greater for delayed than for immediate judgments. Experiment 2 ruled out a plausible artifactual interpretation for accessibility effects on delayed judgments. In the discussion, we explore why study-judgment lags moderate accessibility effects.
\end{abstract}

How do students judge their learning of text materials? For more than two decades, metacomprehension research has focused on this question (Maki, 1998b; Weaver, Bryant, \& Burns, 1995). To investigate metacomprehension judgments, researchers have used a relatively standard method. A participant reads multiple paragraphs, and at some point after reading each one, he or she judges how well he or she will perform on a test about the content of each paragraph. Judgments are often made on a 100-point confidence scale (0-100), with higher judgments indicating more confidence in performing well on the test. Researchers have sought to discover cues that are the basis of these metacomprehension judgments, such as domain familiarity and processing ease. In the present research, we investigate the influence of one cue - momentary accessibility - that is believed to have a substantial influence on these judgments, even though its influence has been empirically validated in only one previous investigation.

Momentary accessibility was first investigated by Morris (1990), who proposed that during the brief time prior to making a judgment, a person recalls as much information about the text as possible. A judgment is then made based on access to the text, with judgments being higher when recalled information is accessed quickly and more information is recalled. To evaluate this momentaryaccessibility hypothesis, Morris measured accessibility by having participants complete a speeded recall trial after making the judgments. At the beginning of each speeded recall trial, participants were prompted with a title of a

We thank Katherine Rawson for helpful comments on a draft of the manuscript. Please send correspondence to J. Dunlosky, P.O. Box 5190, Psychology Department, Kent State University, Kent, OH 44242 (e-mail: jdunlosk@kent.edu). given text. After the title was presented, participants had a brief window of opportunity ( $15 \mathrm{sec}$ or $20 \mathrm{sec}$ in Experiments 1 and 2, respectively) to recall as much as possible from the corresponding text. Two measures of access were then computed. Access latency was the time between onset of the title and a participant's first utterance, and recall was the number of content words recalled in the given window. As predicted by the hypothesis, judgments were negatively correlated with access latency, so that faster latencies were associated with numerically higher judgments, and judgments were positively correlated with recall, so that recalling more words was associated with higher judgments.

Although the experiments reported here may be the first ones inspired by Morris's (1990) momentary-accessibility hypothesis, the effects of accessibility on metacomprehension described by Morris appear to be well-received generalizations within the field (see, e.g., Koriat, 1993; Maki, 1998a; Rawson \& Dunlosky, 2002; Sjostrom \& Marks, 1994; Thiede \& Anderson, 2003). Most importantly, we know of no published research that has replicated Morris's original work or that has assessed the scope of the most relevant effects. The latter is particularly troublesome, because Morris used an uncommon procedure to investigate metacomprehension judgments. Whereas the majority of studies have examined judgments that are made immediately after reading, in Morris's research, the judgments were delayed $24 \mathrm{~h}$ after reading.

Delaying judgments in this manner may be necessary for obtaining accessibility effects, which may not even arise for immediate judgments. In particular, several plausible arguments imply that momentary access will have a substantially greater influence on delayed than on immediate judgments. First, immediately after reading, each text may be quickly accessed from short-term memory or long-term 
working memory (Ericsson \& Kintsch, 1995), and hence, retrieval access immediately after reading may demonstrate little variability across texts. If so, access would not provide a discriminative cue for immediate judgments. Second, other cues, such as domain familiarity and processing ease (Maki, 1998a; Rawson \& Dunlosky, 2002), are known to influence judgments that are made immediately after reading, and these cues may in part dominate immediate judgments. By contrast, when participants are prompted by a text title after a 24-h delay, it seems inevitable that they would attempt to retrieve the originally presented material before making a judgment, which would ensure that momentary access influences judgments at longer lags. For these reasons, we expected the relationship between momentary accessibility and metacomprehension judgments to increase from immediate to delayed judgments. For immediate judgments, accessibility effects may be minimal, which would greatly limit the scope of the momentaryaccessibility hypothesis.

In Experiment 1, we evaluated the momentaryaccessibility hypothesis for two study-judgment lags. We included delayed judgments, to replicate Morris (1990), and used a critical extension of his method to estimate the influence of momentary accessibility on immediate judgments. To foreshadow our results, accessibility was more highly related to delayed than to immediate judgments. Given the potential importance of momentary accessibility to delayed judgments, in Experiment 2, we further scrutinized whether accessibility influences delayed judgments by examining the contribution of a confound - task order (judgments first vs. speeded recall first) — to accessibility effects.

\section{EXPERIMENT 1}

\section{Method \\ Participants and Design \\ Sixty-two undergraduates participated to partially fulfill course requirements in introductory psychology. There were two study- judgment lag (immediate vs. delayed) groups. Thirty participants served in the immediate group and 32 served in the delayed group.}

\footnotetext{
Materials and Apparatus

We used eight short texts and corresponding multiple-choice questions taken from Rawson and Dunlosky (2002, Experiment 3). These texts addressed different topics. The texts varied with respect to intersentential coherence (for details, see Rawson \& Dunlosky, 2002) and were used to ensure that variability occurred in access measures across texts.

All materials were presented to the participants via iMac computers. Verbal outputs from speeded recall trials were recorded onto audiocassette tapes. Computer beeps signaled onset and completion of each speeded recall trial.

\section{Procedure}

The participants were tested individually. They were instructed to read each text carefully in preparation for a series of test questions. For each study trial, a text title was initially presented alone for $2 \mathrm{sec}$ and was followed by the presentation of the entire text. Reading times were recorded by the computer. The texts were presented in random order for each participant, with the initial order being preserved throughout the remainder of the tasks for any given
}

participant. When the participants made judgments (see below for the schedules of judgments for each group), the title of the text was presented, along with the question: "How certain are you that you will be able to correctly answer questions about this text in approximately 10 minutes? 0 (definitely unable) . . 100 (definitely able)."

In the speeded recall task, the participants were instructed that each title would appear individually on the screen, and that they were to begin recalling content from the paragraph out loud as quickly as possible when the title appeared on the screen. Each speeded recall trial lasted $20 \mathrm{sec}$ (as in Morris, 1990, Experiment 2) and was separated by a brief $(5-\mathrm{sec})$ delay with a reminder to prepare for the next trial. The participants were not forced to respond and hence could omit responding if they failed to remember content from the text.

Following the judgment and speeded recall tasks, the participants were given the 8 five-alternative test questions for each text. Test questions tapped information that was either explicitly stated in a text or could be inferred from it. The questions were blocked according to text.

Task order for the experimental groups differed slightly. For the immediate group, each text was presented individually, and immediately following each text, the participants made their judgment about it. Each judgment was followed by the speeded recall trial for that text. The participants completed the same cycle until all the texts had been studied, judged, and recalled. After this, the participants completed the multiple-choice questions.

The participants in the delayed group read all texts on Day 1 and returned to complete the rest of the tasks $24 \mathrm{~h}$ later. They started Day 2 by first making a judgment about each text (as in Morris, 1990). After completing all the judgments, the participants completed the speeded recall task for each text. Finally, the participants completed the multiple-choice questions. ${ }^{1}$

\section{Data Coding}

Transcriptions of the speeded recall data were scored for text content as in Morris (1990). Namely, content words were defined as "all words except $a$, an, and the, with contractions and the not in cannot counted as separate words" (p. 225). Metacognitive statements were also excluded when scoring recall content (e.g., "I don't quite remember this one" or "This paragraph was a tough one"). To measure access latencies (for trials in which text content had been recalled), three raters independently listened to the recorded responses and timed (with a stopwatch) the interval between the presentation of each title and the participant's first utterance. The mean across the three latencies was computed and was used as the measure of access latency for each text.

\section{Results \\ All effects reported as reliable have $p<.05$. \\ Relations Between Measures of Momentary Accessibility and Judgments}

Momentary accessibility was operationalized as follows: (1) access latency was measured (in seconds) from the onset of the title prompt until the first vocal utterance of a participant, and (2) recall was computed by counting the number of content words in each recall protocol. Note that analyses involving access latencies were based only on trials in which the participants recalled text content. Thus, analyses of access latencies could not be influenced by failures to access text content, ${ }^{2}$ and they therefore provide the most relevant estimate of access latency effects on people's judgments.

Analyses reported below were conducted by first computing a gamma correlation between each measure of accessibility and judgments for each participant (Nel- 
son, 1984) and then computing the mean value across participants.

Access latency. The mean correlations involving access latency are reported separately for the two groups in the left panel of Figure 1. To evaluate whether access latencies were a basis for the judgments, we compared the correlations to zero. The correlation for the immediate group did not differ from zero $[t(29)=0.12]$, whereas it did reliably differ from zero for the delayed group $[t(29)=$ 6.04]. As predicted, this relationship was reliably larger for the delayed than for the immediate group $[t(58)=3.40$, effect size $(E S)=0.87]$.

Recall. The mean correlations involving recall are presented in the right panel of Figure 1. Correlations reliably differed from zero for the immediate group $[t(29)=2.23]$ and for the delayed group $[t(29)=8.30]$. This relationship was reliably stronger for the delayed than for the immediate group $[t(58)=2.89, E S=0.76]$.

A secondary issue here is whether the information recalled during each speeded recall trial was from the corresponding text; that is, did people correctly recall information about the text? When the participants recalled text content, nearly all speeded recall trials contained correct information that was from the relevant text. Indeed, only one recall trial (from a single participant) contained information that was not from the corresponding text. Thus, recall of incorrect information evidently did not contribute to the accessibility effects shown in Figure 1.

\section{Mean Access Latencies and Judgment Latencies}

Another consideration in evaluating the momentaryaccessibility hypothesis is whether the participants would have had enough time to access text information prior to making each judgment. Given how quickly participants often make these judgments, one possibility is that access latencies are longer than judgment latencies, which would suggest that the access-judgment correlations (Figure 1) are a result of a third factor and would provide strong evidence against the hypothesis. However, if the participants access the text in the moments prior to making judgments, judgment latencies will be longer than access latencies.

The mean across participants' median latencies to make judgments and to access texts (during speeded recall) are reported in Table 1 . A 2 (judgment lag) $\times 2$ (latency type: access vs. judgment) ANOVA was conducted. As is evident from inspection of Table 1, access latencies (and corresponding judgment latencies) increased as the judgment lag became longer $\left[F(1,60)=19.8, M S_{\mathrm{e}}=\right.$ $4.14, E S=0.57]$, indicating that the text content was less readily available from memory a day after reading than immediately after reading. Most important, judgment latencies were reliably longer than were access latencies $\left[F(1,60)=135.0, M S_{\mathrm{e}}=4.20, E S=1.48\right]$. The interaction was also reliable $\left[F(1,60)=7.46, M S_{\mathrm{e}}=4.20, E S=\right.$ $0.60]$, indicating larger differences in latencies between judgment and access at the long delay than immediately after study. Note, however, that latencies were longer for
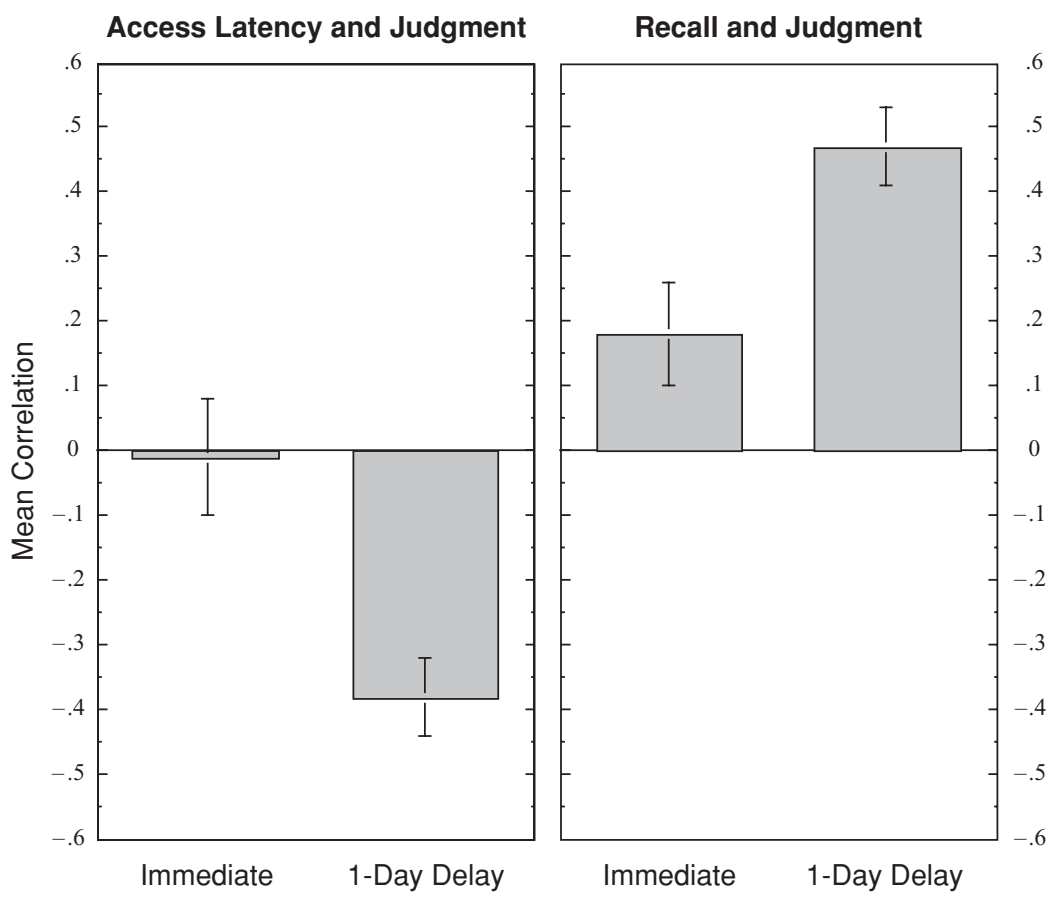

Figure 1. Experiment 1 results. Correlations represent individual gamma correlations between access latency and judgments (left panel) and between recall from speeded recall trials and judgments (right panel). Bars are means across individual gamma correlations, shown with standard errors of the mean. Immediate, judgment made immediately after reading; 1-Day Delay, judgment made a day after initial reading. 
Table 1 Access Latencies and Judgment Latencies

\begin{tabular}{|c|c|c|c|c|}
\hline & \multicolumn{2}{|c|}{ Access Latency (sec) } & \multicolumn{2}{|c|}{ Judgment Latency (sec) } \\
\hline & $M$ & $S E$ & $M$ & $S E$ \\
\hline \multicolumn{5}{|l|}{ Experiment 1} \\
\hline Immediate & 1.44 & 0.33 & 4.92 & 0.49 \\
\hline Long delay & 2.55 & 0.32 & 8.19 & 0.47 \\
\hline \multicolumn{5}{|l|}{ Experiment 2} \\
\hline Recall-judge & 1.94 & 0.13 & 5.36 & 0.56 \\
\hline Judge-recall & 2.06 & 0.16 & 6.78 & 0.51 \\
\hline
\end{tabular}

Note-Values are means across individuals' median access latency and judgment latency. In Experiment 2, all judgments were delayed $24 \mathrm{~h}$ after reading. $S E$, standard error of the mean.

judgments than for recall access for immediate judgments $[t(58)=6.28, E S=1.62]$ and for delayed judgments $[t(62)=10.2, E S=2.56]$.

\section{Test Performance and Judgment Accuracy}

Although test performance and judgment accuracy were not relevant to evaluating the momentary-accessibility hypothesis, we included them for archival purposes because these measures are often reported in the literature. The mean number of questions answered correctly per text for the immediate judgment group $(M=3.8, S E=$ $0.25)$ and the delayed judgment group $(M=3.4, S E=$ $0.16)$ did not reliably differ $[t(60)=1.34]$.

For relative accuracy of the judgments, we computed a gamma correlation between each participant's judgments and his or her own test performance (for the rationale, see Nelson, 1984). As reported in Table 2, accuracy was quite low-as is the case for metacomprehension accuracy in general (Maki, 1998a; Morris, 1990; for an exception, see Thiede \& Anderson, 2003) — and did not differ between the groups $[t(58)=0.44]$.

\section{EXPERIMENT 2}

Experiment 1 demonstrated the need to consider study-judgment lag when investigating the influence of momentary accessibility on judgments, because these effects may be most evident in situations in which people's judgments are delayed. More specifically, although an accessibility effect was obtained for immediate judgments (right panel of Figure 1), these effects were substantially larger (and consistently reliable) for delayed judgments. A question still remains, however, whether these relationships indicate that momentary accessibility influences delayed judgments. By having participants make judgments prior to speeded recall, reactive effects of the former may increase the likelihood of obtaining evidence that conforms to the predictions of the momentary-accessibility hypothesis. For instance, when judgments are made first, an individual may control his or her retrieval during the speeded recall task in a manner that aligns access with the judgments. In the present case, when participants make a lower judgment (as opposed to a higher one), they may subsequently put less effort into retrieval, which in turn may lead to slower access latencies and less recall pro- duction. Some evidence from Morris (1990) is relevant to this issue, because he demonstrated qualitatively similar effects regardless of whether judgments (or speeded recall trials) occurred first. However, these two task orders were manipulated across experiments, which also differed procedurally in other ways, making conclusions from any comparisons between them tenuous. Given the importance of establishing momentary access as a basis of delayed judgments, and to further replicate critical outcomes from Morris, we examined this issue by manipulating task order for delayed judgments in a single experiment.

In Experiment 2, both groups made delayed judgments. One group (judge-recall group) made judgments first, as in Experiment 1, whereas the other group (recall-judge group) had the speeded recall task first. This design affords the critical comparison involving correlations between judgments and measures of access. According to the momentary-accessibility hypothesis, regardless of task order, accessibility effects will be evident, and response latencies should be consistently faster for recall access than for judgments.

\section{Method}

\section{Participants and Design}

Sixty-four undergraduates participated to partially fulfill course requirements in introductory psychology. The experimental design consisted of task order (recall-judge vs. judge-recall) as a betweenparticipants factor. Thirty-three participants served in the recalljudge group and 31 served in the judge-recall group.

\section{Materials and Procedure}

All materials were the same as in Experiment 1. The only difference in procedures across experiments involved the recall-judge group, in which the speeded recall task was completed before the judgment task.

\section{Results}

\section{Relations Between Measures of Momentary Accessibility and Judgments}

As is evident from inspection of Figure 2, delayed judgments demonstrated accessibility effects regardless of task order, and the effects were also similar in magnitude. These observations were supported by the following inferential tests, which provide strong support for the momentaryaccessibility hypothesis for delayed judgments.

Access latency. The mean correlations between access latencies and judgments for the two groups are presented in Figure 2. Correlations from both groups reliably dif-

Table 2

Relative Accuracy of the Metacomprehension Judgments

\begin{tabular}{clcc}
\hline \multirow{2}{*}{ Experiment 1 } & Immediate & $M$ & $S E$ \\
\hline \multirow{2}{*}{ Experiment 2 } & Long delay & .06 & .07 \\
& Recall-judge & .10 & .07 \\
& Judge-recall & .18 & .07 \\
\hline
\end{tabular}

Note-Values are means across individuals' gamma correlations between judgments and test performance. In Experiment 2, all judgments were delayed $24 \mathrm{~h}$ after reading. $S E$, standard error of the mean. 

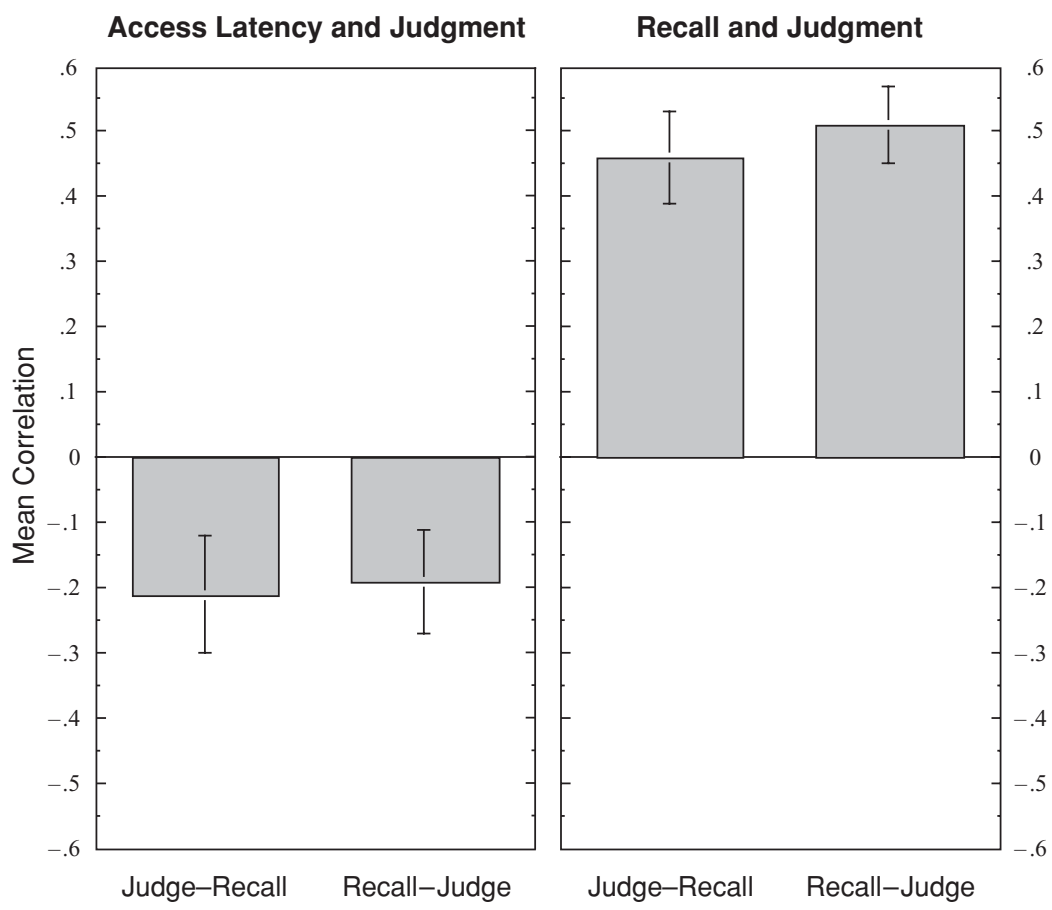

Figure 2. Experiment 2 results. Correlations represent individual gamma correlations between access latency and judgments (left panel) and between recall from speeded recall trials and judgments (right panel). Bars are means across individual gamma correlations, shown with standard errors of the mean. Judge-Recall, group that made judgments before speeded recall trials; Recall-Judge, group that had speeded recall trials before making judgments.

fered from zero [recall-judge group, $t(32)=8.98$; judgerecall group, $t(29)=7.08$ ] but did not reliably differ from each other $[t(61)=0.55]$.

Recall. The mean correlations between recall production and judgments for the two groups are presented in Figure 2. Correlations from both groups reliably differed from zero [recall-judge group, $t(31)=2.36$; judgerecall group, $t(28)=2.45]$ but did not reliably differ from each other $[t(59)=0.13]$.

As in Experiment 1, we also examined the content of recalled information during the speeded recall trials. Again, when participants responded, the information recalled was always - that is, on all trials - relevant to the corresponding text content. Thus, recall of irrelevant (incorrect) information could have no bearing on the accessibility effects presented in Figure 2.

\section{Mean Access Latencies and Judgment Latencies}

Judgment latencies and access latencies were computed as in Experiment 1 and are presented in Table 1. A 2 (task order) $\times 2$ (latency type) ANOVA revealed a main effect for latency type $\left[F(1,62)=103.7, M S_{\mathrm{e}}=5.1\right.$, $E S=1.27]$, indicating that response latencies were slower for judgments than for recall access. The main effect of task order approached reliability $\left[F(1,62)=3.91, M S_{\mathrm{e}}=\right.$ $4.83, p=.052, E S=0.25]$, revealing a trend of faster latencies for both judgments and recall access when the speeded recall task occurred first. The interaction was not reliable $\left[F(1,62)=2.6, M S_{\mathrm{e}}=5.1\right]$.

\section{Test Performance and Judgment Accuracy}

As in Experiment 1, we reported test performance and judgment accuracy for archival purposes. The mean numbers of questions answered correctly per text for the recalljudge group $(M=3.8, S E=0.15)$ and the judge-recall group $(M=3.3, S E=0.14)$ reliably differed $[t(62)=$ 2.27]. Accuracy (Table 2) again was poor and did not differ reliably between groups $[t(61)=0.76]$.

\section{DISCUSSION}

In the present experiments, we provide a critical replication and extension of outcomes originally reported by Morris (1990). First, our findings do replicate those from the original work. Delayed judgments were related (as predicted) to access latency and to recall from speeded recall trials, regardless of the order of the judgments and speeded recall trials. Response latencies were consistently faster for initial access of the texts than for judgments, suggesting that participants had enough time to access text content before making judgments. A more critical issue for the present research pertained to whether accessibility effects would also occur for immediate judgments, which to date have received the majority of attention in 
the field. For these judgments, a reliable relationship was obtained with recall production, which demonstrates that momentary accessibility can influence judgments regardless of the study-judgment lag. Nevertheless, in comparison to delayed judgments, accessibility effects were minimized for immediate judgments, with the correlation between immediate judgments and access latencies reduced to zero.

One plausible explanation for these outcomes concerns the degree to which momentary access discriminates between texts. When participants made immediate judgments, as opposed to delayed judgments, text content may have been readily accessible and may not have been as discriminative. Consistent with this prediction, access latencies were faster for the immediate than for the delayed group (Table 1). Although informative, this comparison does not reveal variability in accessing text content at the level of individual participants, which may be reduced for access measured immediately after study when compared with that measured after a longer delay. To evaluate this prediction, we computed the standard deviation of both measures of accessibility separately for each participant. The mean across participants' standard deviations are presented (for both experiments) in Table 3 . As predicted, intraindividual variability was less for participants who made immediate as opposed to delayed judgments. This trend was reliable for recall $[t(59)=6.75, E S=1.73]$, but was not statistically reliable for access latency $[t(60)=$ $1.02]$. Thus, reduced variability in momentary access immediately after reading may be partially responsible for its diminished influence on immediate judgments, especially for recall production.

In conclusion, regardless of the ultimate explanation for why study-judgment lags moderate accessibility effects (Figure 1), researchers should be cautious not to overgeneralize the influence of momentary accessibility to conditions other than those involving longer study-judgment lags. Given this conclusion, this research also highlights the potential benefits of comparing outcomes from multiple study-judgment lags (cf. Maki, 1998a). By comparing such outcomes, we will achieve a better understanding of the shifting bases of people's metacomprehension

Table 3

Intraindividual Standard Deviations of Momentary Access Measures

\begin{tabular}{cccccc}
\hline & \multicolumn{2}{c}{ Latency $(\mathrm{sec})$} & & \multicolumn{2}{c}{ Recall (words) } \\
\cline { 2 - 5 } & $M$ & $S E$ & & $M$ & $S E$ \\
\hline Experiment 1 & & & & \\
Immediate & 0.92 & 0.20 & & 0.99 & 0.52 \\
$\quad$ Long delay & 2.14 & 1.14 & 15.79 & 0.85 \\
Experiment 2 & & & & \\
$\quad$ Judge-recall & 1.12 & 0.17 & 16.53 & 0.88 \\
Recall-judge & 1.31 & 0.22 & 14.42 & 0.81 \\
\hline
\end{tabular}

Note-Values are means across individuals' standard deviations for the two measures of access. In Experiment 2, all judgments were delayed $24 \mathrm{~h}$ after reading. $S E$, standard error of the mean. judgments at various lags, which are all relevant to how individuals may evaluate their learning of text materials.

\section{REFERENCES}

ERICSSON, K. A., \& KINTSCH, W. (1995). Long-term working memory. Psychological Review, 102, 211-245.

Koriat, A. (1993). How do we know that we know? The accessibility model of the feeling of knowing. Psychological Review, 100, 609639.

MAKI, R. H. (1998a). Predicting performance on text: Delayed versus immediate predictions and tests. Memory \& Cognition, 26, 959-964.

MAKI, R. H. (1998b). Test predictions over text material. In D. J. Hacker, J. Dunlosky, \& A. C. Graesser (Eds.), Metacognition in educational theory and practice (Vol. 14, pp. 117-144). Mahwah, NJ: Erlbaum.

MorRIS, C. C. (1990). Retrieval processes underlying confidence in comprehension judgments. Journal of Experimental Psychology: Learning, Memory, \& Cognition, 16, 223-232.

Nelson, T. O. (1984). A comparison of current measures of the accuracy of feeling-of-knowing predictions. Psychological Bulletin, 95, 109-133.

Rawson, K. A., \& Dunlosky, J. (2002). Are performance predictions for text based on ease of processing? Journal of Experimental Psychology: Learning, Memory, \& Cognition, 28, 69-80.

Sjostrom, K. P., \& Marks, A. (1994). Pretest and posttest confidence ratings in test performance by low-, medium-, and high-scoring students. Teaching of Psychology, 21, 12-16.

Son, L. K., \& Metcalfe, J. (2005). Judgments of learning: Evidence for a two-stage process. Memory \& Cognition, 33, 1116-1129.

Thiede, K. W., \& ANDERson, M. C. M. (2003). Summarizing can improve metacomprehension accuracy. Contemporary Educational Psychology, 28, 129-160.

Weaver, C. A., III, Bryant, D. S., \& Burns, K. D. (1995). Comprehension monitoring: Extensions of the Kintsch and van Dijk model. In C. A. Weaver III, S. Mannes, \& C. R. Fletcher (Eds.), Discourse comprehension: Essays in honor of Walter Kintsch (pp. 177-193). Hillsdale, NJ: Erlbaum.

\section{NOTES}

1. As a result of this design, the immediate and delayed groups differed with respect to whether or not judgments and the speeded recall trials were interspersed. We chose this particular design for two reasons. First, we wanted to use Morris's (1990) procedure - in which delayed judgments and speeded recall trials were conducted in separate blocks - to most closely replicate the original accessibility effects. Second, although we could have blocked all speeded recall trials after immediate judgments had been made and all texts had been read, blocking trials in this way would have reduced the likelihood of demonstrating accessibility effects for immediate judgments, because the measure of access would have been delayed and hence might not reflect access at the time of the immediate judgments. Accordingly, we chose to measure momentary access immediately after each judgment was made, in order to explore accessibility effects under conditions that were most likely to produce them.

2. For trials in which content was not recalled, there is an issue concerning the degree to which judgments are influenced by the latency of responding that one does not remember any text content. For instance, quickly stating "I don't know" may lead to low metacomprehension judgments (as it does with judgments of learning for paired associates; Son \& Metcalfe, 2005). Although this is an interesting issue, we did not design the procedure to validly measure latencies of responding "do not know" during the speeded recall task, and such latencies were not relevant to evaluating the momentary-accessibility hypothesis. Accordingly, this particular issue cannot be addressed in the present research.

(Manuscript received March 2, 2005; revision accepted for publication May 23, 2005.) 